\title{
OBESITY INCREASES THE RISK OF BREAST CANCER IN POSTMENOPAUSAL WOMEN?
}

Carolina R. Mendonça', Magno B. Cirqueira', Jalsi T. Arruda², Nilza N. Guimarães³, Erika A. Silveira1', Waldemar N. do Amaral ${ }^{1}$

1Programa de Pós-Graduação em Ciências da Saúde, Universidade Federal de Goiás - Goiânia (GO), Brazil.

²Faculdade de Medicina, Centro Universitário de Anápolis (UniEVANGÉLICA) - Anápolis (GO), Brazil.

${ }^{3}$ Departamento de Morfologia, Instituto de Ciências Biológicas, Universidade Federal de Goiás - Goiânia (GO), Brazil.

Objective: To describe the impact of obesity on the increased risk of breast cancer in postmenopausal women. Methodology: The search for scientific articles was carried out in the databases: PubMed and Scopus. Articles published in English in the last five years have been selected. The surveys were conducted with the following combinations: ("Obesity"[MeSH Terms] AND "Breast Neoplasms" [MeSH Terms] AND "postmenopausal women" [All Fields]). Criteria for definition of obesity was the Body Mass Index (BMI) $\geq 30$. Results: We included 25 studies. Most epidemiological studies report that women with obesity and overweight are at higher risk of developing postmenopausal breast cancer. BMI $\geq 30$, at the time of diagnosis, is associated with more advanced stages of breast cancer with more aggressive tumor characteristics, larger tumor size and worse histological grade. An increase of 5 units in BMI is associated with a $12 \%$ increased risk of breast cancer. Among postmenopausal women, obesity has a $20 \%$ to $40 \%$ increased risk of developing breast cancer compared to women of normal weight. The greatest risks are observed mainly in women who have never used menopausal hormone therapy. Weight gain after diagnosis indicated an increase in mortality rates and obesity would be linked to an increased risk of secondary neoplasms. Obese women compared to normal weight women had a 21-41\% higher overall mortality risk and a 25-68\% risk of breast cancer mortality, depending on how much time had elapsed since the diagnosis of the disease. Another study suggests that increasing body fat percentage 6 months after breast cancer surgery is an important predictor of post-menopausal distant metastases. In another study, high BMI was protective in premenopausal women ( $\mathrm{OR}=0.14 \mathrm{per}$; $95 \% \mathrm{CI} 0.02-0.77)$, while in postmenopausal women it was a risk factor for development of breast cancer ( $\mathrm{OR}=2.39$ per; $95 \% \mathrm{CI} 1.02-5.55)$. One explanation for the increased risk is that obesity leads to the release of cytokines into the bloodstream that affect the metabolism of breast cancer cells, making them more aggressive. Further, the impact of obesity on risk differs by tumor hormone receptors status and, among postmenopausal women, use of hormone replacement therapy. Conclusion: Obesity is associated with an increased risk of postmenopausal breast cancer. 